\title{
Nanopore Sequencing as a Surveillance Tool for Plant Pathogens in Plant and Insect Tissues
}

\author{
Aline Bronzato Badial, Department of Biochemistry, Molecular Biology, Entomology and Plant Pathology, Mississippi State University, \\ Starkville, MS; Diana Sherman and Andrew Stone, USDA-ARS Foreign Disease-Weed Science Research Unit, Fort Detrick, MD; Anagha \\ Gopakumar and Victoria Wilson, Department of Biochemistry, Molecular Biology, Entomology and Plant Pathology, Mississippi State Uni- \\ versity, Starkville, MS; William Schneider, USDA-ARS Foreign Disease-Weed Science Research Unit, Fort Detrick, MD; and Jonas King, ${ }^{\dagger}$ \\ Department of Biochemistry, Molecular Biology, Entomology and Plant Pathology, Mississippi State University, Starkville, MS
}

\begin{abstract}
Plant pathogens are constantly emerging and spreading into new areas and there are often limited postdiagnosis treatment options for infection, making surveillance key to their control. Here we present results from a study testing the efficacy of a portable nanopore-based massively parallel sequencing (MPS) technology for use in the detection of diverse plant pathogens in selected samples. The Oxford MinION device was coupled with whole transcriptome amplification (WTA) to sequence the metatranscriptome of plant and insect tissues infected with either Candidatus Liberibacter asiaticus or plum pox virus. Results showed that this methodology is useful for detecting

unsuspected viral and bacterial pathogens in plant and insect tissues. The percentage of generated reads assigned to plum pox virus was $95 \%$ from infected tissue and 3\% from the viruliferous insect, Myzus persicae. Diaphorina citri sequencing led to $22 \%$ of the reads mapping as $\mathrm{Ca}$. L. asiaticus. Plum pox virus and $C a$. L. asiaticus were detected in both tissue and insect samples near the beginning of each sequencing run, demonstrating the capability of this methodology to obtain results rapidly. This approach also proved the capability of this system to determine the major components of the insect vector's microbiome and the specific strain of small-genome, high-titer pathogens.
\end{abstract}

As the global population steadily increases and the threat of climate change rises, our ability to combat plant pathogens promises to become an important factor in the fight for food security. For many plant pathogens, there are few postexposure options for intervention. As a result, the detection of the pathogen plays a key role in containing their spread. Although several technologies exist for molecular detection of plant pathogens, nearly all currently feasible methods require previous knowledge of the pathogen involved, i.e., the assays are inherently targeted against specific pathogens. Recently, massively parallel sequencing (MPS), or next-generation sequencing, has been shown to be useful in identifying pathogens that are not anticipated to be in the sample, or that may even be unknown to science (Adams et al. 2009; Stobbe et al. 2013). While this approach is promising, two main hurdles limit its use in remote settings: 1) the sequencing hardware is not portable, and 2) querying the sequences produced can require sophisticated bioinformatics and timely analyses of a substantial dataset may require high performance computational hardware. Several studies have shown that techniques such as electronic-probe diagnostic nucleic acid analysis, or EDNA, can be used to overcome the second hurdle mentioned above. This technique minimizes and ignores irrelevant sequence data and thereby focuses on specific pathogen-associated sequences (Espindola et al. 2015; Stobbe et al. 2013, 2014).

To assess one possible means of overcoming the lack of equipment portability, we tested the effectiveness of the first portable MPS device as a surveillance tool for detection of viral and bacterial plant pathogens in both infected plant and insect tissues. Specifically, an Oxford Nanopore Technology MinION sequencer was used (Oxford, U.K.). MinION is a portable, single molecule genome-sequencing

${ }^{\dagger}$ Corresponding author: J. King; E-mail: jonas.king@msstate.edu

Funding: National Institute of Food and Agriculture, U.S. Department of Agriculture (1008525); Foundation for Food and Agriculture Research (534275).

Any opinions, findings, conclusions, or recommendations expressed in this publication are those of the author(s) and do not necessarily reflect the view of the U.S. Department of Agriculture.

Accepted for publication 5 March 2018.

C) 2018 The American Phytopathological Society instrument that works by taking frequent electrical measurements while a single strand of DNA passes through a protein nanopore. This device is the size of a smart phone, is USB powered, and can stream up to $100 \mathrm{MB}$ of sequence data over a 24-h period. The library preparation is also uncomplicated, and the whole protocol can be executed in less time than when using other comparable MPS technologies. This system was previously demonstrated to be promising for surveillance of viral and bacterial pathogens of humans (Kilianski et al. 2015; Quick et al. 2014, 2015), and single-nucleotide-variant detection in clonal haploid samples (Jain et al. 2015). In order to simulate a diversity of plant pathogens for this initial study, a viral and a bacterial plant pathogen that are each vectored by hemipteran insects were selected. Plum pox virus (PPV) is a major viral pathogen of stone fruit around the world (García et al. 2014). For PPV, we tested both infected plant and insect samples in separate trials. The second pathogen is Candidatus Liberibacter asiaticus, the causative agent of citrus greening, the most destructive citrus pathogen worldwide. As Diaphorina citri is the principal insect vector responsible for infection and spread of this disease and insect surveillance is a major factor in monitoring citrus greening's spread, we decided to focus on the infected insect for this experiment (Gottwald 2010; Ukuda-Hosokawa et al. 2015). This insect was also selected because its bacterial microbiome is well known, and it was therefore simple to assemble a reference dataset that included all predicted bacterial genomes (Fagen et al. 2012). We also developed a computational pipeline for pathogen detection; specifically, querying error-prone MinION output against genome-based reference datasets. This pipeline uses currently available open source software packages to base-call, align, and conduct statistical analyses on these data.

Our results suggest that the combination of whole transcriptome amplification (WTA) and nanopore sequencing technologies, or a similar derivative, could be used to detect emergent plant pathogens from infected tissues and also be adapted for use in assaying beneficial plant-associated microbiomes. The speed at which this technology was able to produce pathogen-confirmatory reads suggests that it could also be downscaled in the future to produce more affordable and portable platforms.

\section{Materials and Methods}

Material. Three experiments were conducted using plant and insect tissues infected with either of two selected plant pathogens, $C a$. L. asiaticus or PPV. The insect vectors, D. citri infected with Ca. L. asiaticus, Myzus persicae carrying PPV, and the fruit Prunus 
persica infected with PPV, were used as models for plant pathogen detection. Samples were obtained from the greenhouse and insectarium rearing facilities at the USDA-ARS, Foreign Disease-Weed Science Research Unit, Fort Detrick, Maryland. M. persicae were reared on Rumex crispus (curly dock) in a virus-free insectary and after 3 weeks of colony initiation insects were fed on PPV-infected $P$. persica (cv. GF305). Non-Ca. L. asiaticus-exposed D. citri were maintained on Murraya koenigii (curry leaf) and at 5 days post molt, adults were then fed on $\mathrm{Ca}$. L. asiaticus-infected Citrus sinensis cv. Pineapple (pineapple sweet orange) for an acquisition access period of 7 days. Plant infection was primarily confirmed via observation of physical symptoms as well as standard PCR analyses.

RNA preparation. After sample collection, nucleic acids were extracted immediately from the tissue samples using an RNeasy Fibrous Tissue Mini Kit (Qiagen, Valencia, CA). Following RNA extraction, total RNA content was amplified using the TransPlex WTA Kit (Sigma Aldrich, St. Louis, MO) following the manufacturer's instructions.

Sample quality control and quantification. Quality and quantification of the WTA samples were conducted at multiple steps during the library preparation by means of a QIAxcel capillary electrophoresis unit (Qiagen, Valencia, CA) and a Qubit fluorimeter (Thermo, Waltham, MA).

MinION library preparation. Library preparation for amplicon sequencing was achieved using an Oxford SQK-MAP006 ligation sequencing kit following the manufacturer's instructions (Oxford Nanopore Technologies, Oxford, U.K.). Briefly, a total of $1 \mu \mathrm{g}$ of D. citri WTA sample, $800 \mathrm{ng}$ of $M$. persicae WTA sample, and $1 \mu \mathrm{g}$ of $P$. persica WTA sample were end-repaired using an NEB end-repair module (New England Biolabs, Ipswich, MA), then cleaned using Agencourt AMPure XP beads (Beckman Coulter, Indianapolis, IN) with $1.8(\mathrm{v} / \mathrm{v})$ ratio of beads to reaction mixture. Endrepaired DNA was A-tailed using the NEB dA-tailing module (New England Biolabs) in a volume of $55 \mu \mathrm{l}$, and again cleaned up with AMPure XP beads as above. Following cleaning, $10 \mu \mathrm{l}$ of the adapter mix and $2 \mu \mathrm{l}$ of the HP adapter (supplied in the SQK-MAP006 library preparation kit, Oxford Nanopore Technologies) were added to the dA-tailed amplicons along with $50 \mu \mathrm{l}$ of the NEB-Blunt/TA ligase master mix (New England Biolabs) and incubated for $10 \mathrm{~min}$. The resulting sequencing library was purified using a solution of Dynabeads MyOne Streptavidin C1 (Life Technologies, Carlsbad, CA). Six microliters of the purified library was mixed with $75 \mu l$ of running buffer, $65 \mu l$ of nuclease-free water, and $4 \mu l$ of fuel mix, and loaded on the flow cell.

MinION sequencing configuration. Each sample was run individually in a different flow cell. The flow cell was first primed using $500 \mu \mathrm{l}$ of the blank buffer solution $(500 \mu \mathrm{l}$ of running buffer, $474 \mu \mathrm{l}$ of nuclease-free water, and $26.6 \mu \mathrm{l}$ of fuel mix) and incubated for $10 \mathrm{~min}$ at room temperature. The priming process was repeated two times before loading the sample. Library mixture $(150 \mu l)$ was loaded into the MinION flowcell. The sequencing runs were initiated running MinKNOW (version 0.49.2.9), and the "NC_48Hr_Sequencing Run_FLO_MIN106_SQK-LSK208.py" script. Read event data were base-called by Metrichor software (version 0.16.37960) using the workflow 2D Basecalling for MAP-006. Each run continued for a total of $24 \mathrm{~h}$.

Data handling. After sequencing, data were collected as fast5 files containing raw electrical signals. After processing through Metrichor (Oxford Nanopore), base-called data were obtained in the form of fast5 files containing metadata. Fasta sequences and read event data, including the strand translocation times, were extracted for further analysis using the $\mathrm{R}$ package poRe (Watson et al. 2014). Fasta sequence data from each sample was combined into a single fasta archive for downstream alignment analysis.

Alignment analysis. Nucleotide base alignment was carried out with GraphMap Aligner (Sović et al. 2016). GraphMap generates sam files, which were converted into bam files using SAMtools software ( $\mathrm{Li}$ et al. 2009). Metadata from each bam file containing the alignment information were analyzed using the $\mathrm{R}$ package Rsamtools (Morgan et al. 2016), Bamstat 1.25, a $\mathrm{C}++$ program developed to tabulate mapping statistics, and the BEDtools software (Quinlan and Hall 2010). True alignment was considered for both positive and negative strands, by following the GraphMap parameters for correct alignment. Each read was assigned with a quality indicator (mapQ), and those reads with a quality score $\geq 40$ were accepted for inferences, while reads with quality rate $\leq 40$ were not considered as a true alignment. All analyses were performed on a standard desktop computer running Linux or Windows depending on software requirements.

Alignments to microbial databases. Each sample dataset was queried against the appropriate portable reference dataset. Reads from $M$. persicae and $P$. persica were tested against the current complete set of high quality, annotated, viral genomes available from NCBI RefSeq (ftp://ftp.ncbi.nlm.nih.gov/refseq/release/ viral). In addition, reads produced from $M$. persicae sample were aligned against a set of four different datasets: the aphid $M$. persicae CDS sequence (M. persicae TGAC clone O v1.1 CDS fasta file) data downloaded from AphidBase (http://bipaa.genouest.org/is/aphidbase/); two genomes of endosymbiotic bacteria known to be major parts of the M. persicae microbiome (Buchnera aphidicola [NZ CP002701] and $\mathrm{Ca}$. Regiella insecticola [GL379589]) (von Burg et al. 2008, Jiang et al. 2013); and the plum pox virus genome (NC_001445.1). Data of the $P$. persica tissue was tested by aligning the produced dataset with the $P$. persica genome (AKXU00000000.2) and the PPV genome (NC_001445.1). The D. citri dataset was analyzed against a collection of seven datasets, including $D$. citri genomic data (gil645508417), the pathogen known to be infecting the food source for the psyllids used in this study $(\mathrm{Ca}$. L. asiaticus NC_020549.1), and five additional genomes from organisms known to comprise the normal endosymbiont bacteria of D. citri (Fagen et al. 2012; Nakabachi et al. 2013) (Candidatus Carsonella rudii [NC_018417.1], Candidatus Profftella armatura [NC_021885.1], Salmonella enterica [NC_003198.1], Klebsiaella variicola [NZ_ JUSW01000023], and Wolbachia wDi [NZ_AMZJ01000037; NZ_KB223535]).

Viral and bacterial detection. To determine the level of pathogen genome coverage and to identify the pathogen present in the samples, the generated reads from each dataset were mapped against the whole reference genome of the respective pathogens. Reads generated from the psyllid $D$. citri were aligned against the genome of $C a$. L. asiaticus (NC_020549.1), while reads from the aphid $M$. persicae and from the fruit $P$. persica were aligned against the PPV genome (NC_001445.1).

\section{Results}

Alignments to microbial databases. Data produced by the MinION was more than sufficient to correctly identify the source pathogens and discriminate among other microbial genomes tested (Table 1; Fig. 1A to C). During the mapping process, all potential alignments were assigned. To represent the datasets in the most unbiased manner (i.e., alignment to several large-genome and highly genome-plastic pandoraviruses), the mean of the reads mapped to a given reference genome was used as a simple summary statistic. The percentage of reads mapped of both $M$. persicae and $P$. persica datasets showed a high number of reads assigned to the PPV reference genome. The mean of reads mapped, adjusted by target genome size, were significantly higher in both plant and insect tissues when compared with all other viral genomes tested (Table 1; Fig. 1A and B). Considering the mean of reads mapped normalized by genome size of the $D$. citri dataset, reads that were assigned to the $C a$. L. asiaticus reference genome showed the third highest percentage of reads mapped (Table 1).

PPV and $\mathrm{Ca}$. L. asiaticus sequencing and detection. The MinION generated reads that confirmed the presence of each of the pathogens tested (Fig. 1D to I). The raw statistics data are described in Table 2. From the three datasets analyzed, numbers of fast 5 reads ranged from 18,960 to 97,906 with a mean read length of 456 to $768 \mathrm{bp}$. A minority of the data output by MinION aligned well to the pathogen reference genomes when reads were mapped solely to these genomes. The number of reads aligned to the pathogen genomes ranged from 968 to 
7,732, with a mean alignment length of 358 to $487 \mathrm{bp}$. Considering mapQ scores $\geq 40$, the total number of reads aligned ranged from 935 to 7,531 (Table 2). The total number of reads aligned with mapQ scores higher than 40 were less than the total number of reads aligned with mapQ lower than 40 . However, for all datasets tested, reads with mapQ $\leq 40$ were produced at the beginning and at the end of the runs, demonstrating the capacity of the MinION to produce reads with favorable mapQ during the entirety of a sequencing run. Despite the total number of high quality aligned reads being lower than the number of reads produced by MinION, the proposed method was able to identify all three target pathogens in the samples tested. Considering only the reads with mapQ scores equaling 40, and the total time of the sequencing run, reads that were correctly mapped with the pathogen targets were clearly produced within the first minute of each run (Fig. 1D to F), which demonstrates the capability of this method to identify pathogens in a fast and reliable way. It should be noted that the data were analyzed immediately following the completion of each run, although there is software that would have made it possible to scan each read during the course of the run. We chose this method because we wanted to rely upon open source analysis software and the more sensitive GraphMap alignment algorithm. In all three host-pathogen systems analyzed here, the total time from the end of each run to the positive identification of each pathogen was less than $2 \mathrm{~h}$ using a standard desktop computer. The first $C a$. L. asiaticus sequence that was positively identified in the $D$. citri sample occurred at $38 \mathrm{~s}$ into the run. Plum pox virus was identified in the $M$. persicae sample at $41 \mathrm{~s}$ into the run, and in the $P$. persica sample at $38 \mathrm{~s}$ into the run. In all cases, the initial matches were quickly confirmed by numerous other reads with positive matches to the pathogen reference genome. The distribution of reads aligned across the known reference genomes is shown in Figure 1 (G-I). The $P$. persica dataset had the largest proportion of reads spanning the reference genome, while reads from the $D$. citri and M. persicae datasets showed lower coverage. The insect samples showed lower genome coverage due to the lower number of total reads. In addition, for Liberabacter, the target organism has a much larger genome than the viral targets, and therefore the lower observed genome coverage was expected.

\section{Discussion}

The development of rapid detection and accurate diagnostic methods for plant pathogens and their vectors plays an important role in fighting the spread of the diseases they cause. The ability to accurately detect multiple pathogens simultaneously is a valuable feature for any diagnostic tool. Regarding vectored pathogens, the ability to detect and discriminate both the pathogen and the vector from a complex background such as an insect trap is highly desirable. Multiplex PCR or immune assays are limited in the number of possible targets to be addressed. In contrast, massively parallel sequencing analysis of metagenomic samples could be used to detect pathogens other than those known to be normally associated with a plant or insect species, provided their titers are high enough. In addition, because RNA viruses are the most common form of plant viruses, the ability to simultaneously detect pathogens with RNA and DNA genomes is highly desirable.

Here we report the first successful use of the Oxford MinION sequencer for use in the detection of plant pathogens from infected plant tissues and insect vectors. In addition, because many plant pathogens such as PPV have RNA genomes, we chose to couple total RNA amplification (WTA) and cDNA library preparation with nanopore analyses. Seeking to simulate a diversity of plant pathogens, a viral and bacterial plant pathogen that are each vectored by hemipteran insects were selected. Although we obtained a high level of reads with low quality score ( $\geq 40$ ), likely due to using WTA samples, pathogen detection was possible using a reference data set that encoded either all available viral genomes or a compilation of bacterial genomes known to associate with the host organisms. Using this approach, it was possible to identify the pathogens in all samples, demonstrating that this method is an effective surveillance tool for plant pathogen detection and can be widely implemented in the future.

Table 1. Raw statistics for alignment generated against a dataset of different genomes using GraphMap aligner

\begin{tabular}{|c|c|c|c|c|c|}
\hline Dataset & Reference genome & $\begin{array}{l}\text { Total of reads } \\
\text { aligned }\end{array}$ & $\begin{array}{c}\text { Reads aligned } \\
\text { mapQ }=40\end{array}$ & $\begin{array}{c}\text { Mean of reads aligned adjusted } \\
\text { to genome size }\end{array}$ & $\begin{array}{c}\text { SD of reads aligned adjusted } \\
\text { to genome size }\end{array}$ \\
\hline \multirow[t]{7}{*}{ Diaphorina citri } & $\begin{array}{l}\text { Candidatus Liberibacter } \\
\text { asiaticus }\end{array}$ & 105 & 13 & 0.022 & 0.265 \\
\hline & D. citri & 3,306 & 1,018 & 0.057 & 0.138 \\
\hline & Carsonella rudii & 10 & 2 & 0.012 & 0.178 \\
\hline & Wolbachia $w D i$ & 27 & 24 & 0.012 & 0.115 \\
\hline & Profftella armatura & 210 & 79 & 0.132 & 2.019 \\
\hline & Klebsiaella variicola & 73 & 25 & 0.016 & 0.104 \\
\hline & Salmonella entérica & 47 & 15 & 0.002 & 0.049 \\
\hline \multirow[t]{3}{*}{ Myzus persicae ${ }^{\mathrm{a}}$} & Apis mellifera virus & 24 & 12 & 0.036 & 0.189 \\
\hline & Mannheimia phage & 80 & 30 & 0.058 & 0.804 \\
\hline & Plum pox virus & 45 & 18 & 0.741 & 0.859 \\
\hline \multirow[t]{4}{*}{ M. persicae $\mathrm{e}^{\mathrm{b}}$} & Plum pox virus & 16 & 15 & 0.739 & 0.828 \\
\hline & Buchnera aphidicola & 07 & 05 & 0.002 & 0.053 \\
\hline & M. persicae & 464 & 130 & 0.136 & 0.292 \\
\hline & $\mathrm{Ca}$. Regiella insecticola & 44 & 12 & 0.047 & 0.15 \\
\hline \multirow[t]{7}{*}{ Prunus persica ${ }^{\mathrm{c}}$} & Plum pox virus & 929 & 889 & 41.721 & 22.027 \\
\hline & Enterobacteria phage & 189 & 186 & 1.616 & 13.699 \\
\hline & $\begin{array}{l}\text { Pandoravirus } \\
\text { inopinatum }\end{array}$ & 254 & 105 & 0.008 & 0.100 \\
\hline & Papiine herpesvirus & 227 & 71 & 0.041 & 0.263 \\
\hline & Mollivirus sibericum & 22 & 11 & 0.011 & 0.104 \\
\hline & A. mellifera virus & 105 & 40 & 0.036 & 0.228 \\
\hline & C. herpesvirus 2 & 96 & 29 & 0.032 & 0.192 \\
\hline \multirow[t]{2}{*}{ P. persica ${ }^{\mathrm{d}}$} & Plum pox virus & 15,372 & 5,652 & 36.286 & 19.933 \\
\hline & P. persica & 807 & 782 & 0.105 & 0.289 \\
\hline
\end{tabular}

${ }^{a} M$. persicae generated dataset tested against the viral reference dataset.

${ }^{\mathrm{b}} M$. persicae generated dataset tested against endosymbiont bacteria of the insect $M$. persicae and PPV reference genomes.

${ }^{c} P$. persica generated dataset tested against the viral reference genome.

${ }^{\mathrm{d}} P$. persica generated dataset tested against $P$. persica and PPV reference genomes. 
A Prunus persica

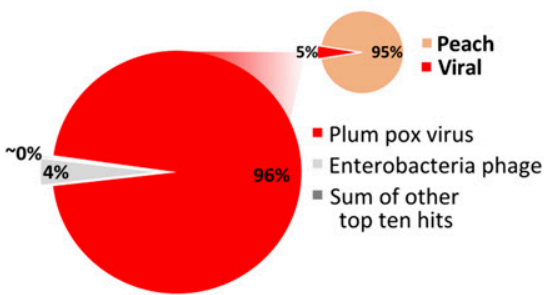

B Myzus persicae

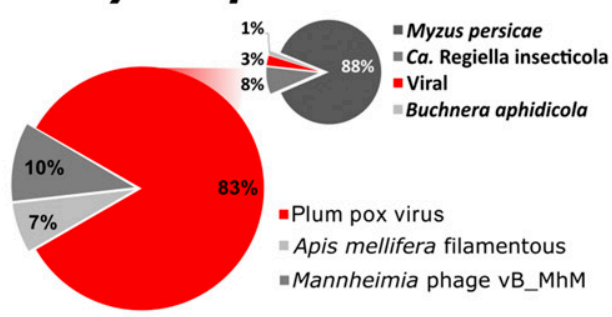

C Diaphorina citri

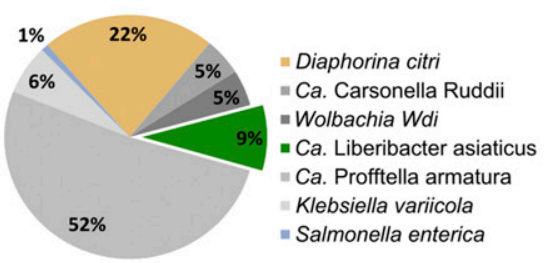

Alignment to microbial databases

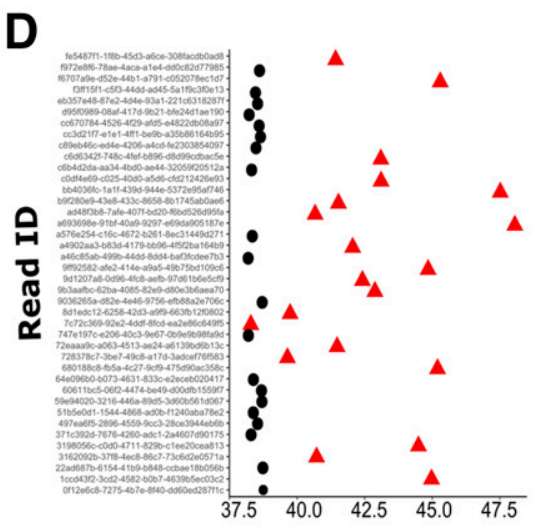

Start Time (seconds)
E

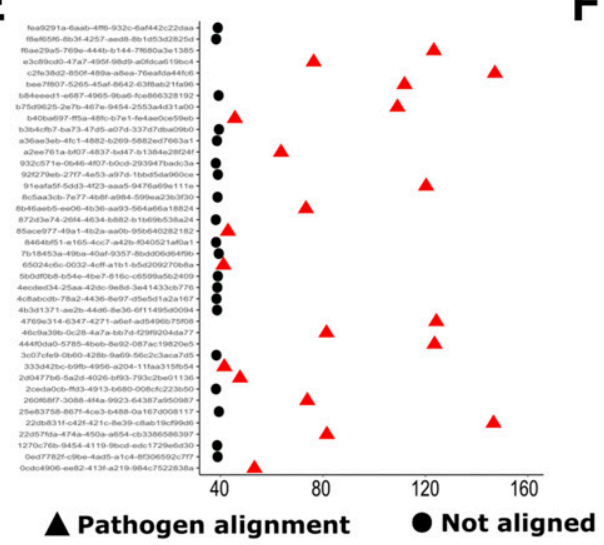

$\mathbf{F}$

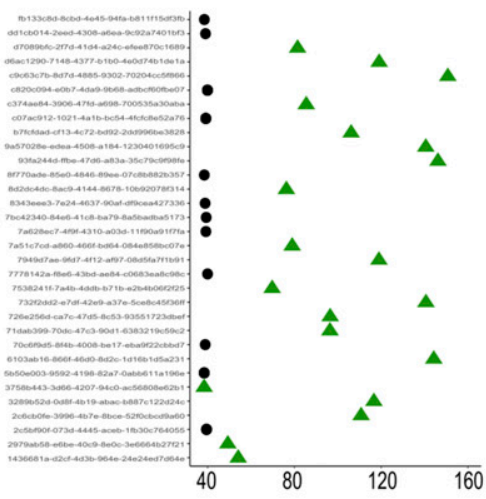

\section{Pathogen detection versus time}

G

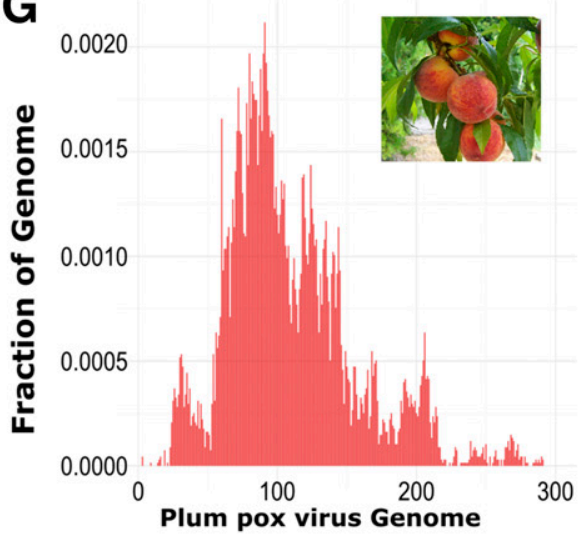

H

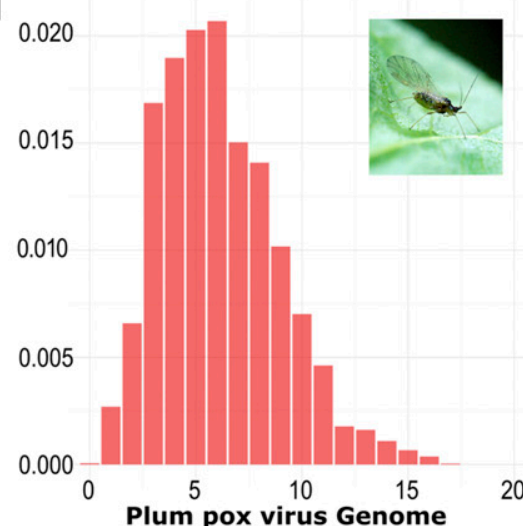

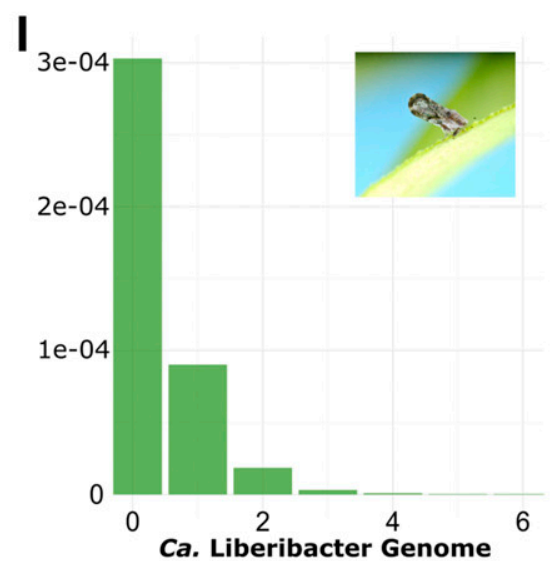

Depth

\section{Reference genome coverage}

Fig. 1. Nanopore sequencing of whole transcriptome amplification is capable of identifying plant pathogens from both plant and insect tissues. A and B, Alignment of reads produced by MinION from Prunus persica and Myzus persicae datasets to the total viral genomes currently available from NCBI RefSeq. The inset in A shows the number of total viral reads versus the number of reads mapping to the $P$. persica genome. The inset in B shows the total number of reads for the insect genome versus viral genomes and the genomes of the two major bacterial endosymbionts of $M$. persicae. C, Alignment of reads from Diaphorina citri dataset aligned to a reference database composed of the known bacterial microbiome of $D$. citri. All data shown in figures from $A, B$, and $C$ were normalized by microorganism genome size. D to $F$, Time of the run that plum pox virus and Candidatus Liberibacter asiaticus were first identified over the $24 \mathrm{~h}$ of the sequencing process. Graphs from $\mathrm{D}$ to $\mathrm{F}$ contain data from the first 20 aligned and unaligned reads to the two pathogens reference genomes. $\mathbf{G}$ to I, Depth of the genome coverage produced by alignments to the whole reference genome of the two pathogens tested here. As expected, the bacterial sample showed lower coverage as the target organism has a much larger genome than the viral target.

Table 2. Raw statistics for alignment generated against the references genomes of plum pox virus and Candidatus Liberibacter asiaticus using GraphMap aligner

\begin{tabular}{lcccccc}
\hline Dataset & $\begin{array}{c}\text { Number of fast5 } \\
\text { reads }\end{array}$ & $\begin{array}{c}\text { Total number of } \\
\text { reads aligned }\end{array}$ & $\begin{array}{c}\text { Min read length } \\
\text { aligned (bp) }\end{array}$ & $\begin{array}{c}\text { Max read length } \\
\text { aligned (bp) }\end{array}$ & $\begin{array}{c}\text { Mean read length } \\
\text { aligned (bp) }\end{array}$ & $\begin{array}{c}\text { Number of reads aligned } \\
\text { mapQ } \geq 40\end{array}$ \\
\hline $\begin{array}{c}\text { Diaphorina } \\
\text { citri }\end{array}$ & 66,705 & 4,701 & 80 & 13,908 & 358 & 2,999 \\
$\begin{array}{c}\text { Myzus } \\
\text { persicae }\end{array}$ & 18,960 & 968 & 83 & 3,163 & 389 & 935 \\
$\begin{array}{c}\text { Prunus } \\
\text { persica }\end{array}$ & 97,906 & 7,732 & 80 & 19,908 & 487 & 7,531 \\
\hline
\end{tabular}


Reads from the $P$. persica dataset showed a large proportion of reads spanning the reference genome. The total number of reads produced for the $P$. persica dataset was also much larger than either insect dataset. There are several reasons to explain why the plantderived dataset produced more total reads and pathogen positive reads. First, it is technically difficult to acquire RNA from these insect samples, and therefore the quality of our RNA input might have been lower for the insect samples. Second, pathogen titers in insects may be proportionally lower than in plants, as the pathogen may be present in some tissues at certain times within an infected insect host. It is known that $\mathrm{Ca}$. Liberibacter is differently distributed in psyllids tissues, with significantly lower titers in the salivary glands than in the rest of the body (Ammar et al. 2011). Fagen et al. (2012) studied the abundance of $C a$. L. asiaticus in the whole insect body of $D$. citri and also found lower levels of this pathogen when compared with four other endosymbiotic bacteria present in the microbiome of the insect. The authors suggested that the variable effect of $\mathrm{Ca}$. L. asiaticus on the endosymbiotic bacteria may be caused by the irregular distribution of $C a$. L. asiaticus within the host causing certain bacteria to be displaced and not others. Potyviruses such as PPV are nonpersistently stylet-transmitted, meaning the only virus present in the insect body are those in the process of moving through the digestive tract. Results from $M$. persicae and $D$. citri datasets from this study are generally in line with these past results, suggesting that the viral and bacterial content in these insects are lower because of the more disperse infection distribution in the insect bodies.

This study shows that nanopore sequencing coupled with WTA, or the development of direct nanopore sequencing of RNA, is a promising alternative means of identifying unexpected pathogens in agricultural settings. The short time in which each assay was able to produce numerous pathogen-confirmatory reads also suggests that the current platform could be improved into an operative format that is more cost-effective and amenable to use in remote settings. Early detection and confirmation of pathogen presence in the sequencing process is advantageous not only because it is more timely, but also because it is extremely useful when data handling is considered, as the large amounts of data usually generated with standard next generation sequencing would potentially be avoided. Oxford Nanopore is constantly upgrading both their flow cells and bioinformatics technologies, and it is expected that this technology will only become more accurate and cost effective in the future. Likewise, Oxford is currently introducing a novel flow cell for the direct sequencing of RNA molecules, which would further streamline our proposed methods. We plan to continue testing this technology in the future by including these and other nanopore-generated data in a cost-benefit analysis of several current MPS platforms and by coupling our nanopore data analysis pipeline with EDNA technology to test for the potential of increased efficiency using basic computational resources (Stobbe et al. 2013). The possibility of eventually downscaling their current platform for more affordable diagnostics will also be explored as the obtained results suggest that a smaller flow cell would likely be effective in the type of scenarios simulated here.

\section{Acknowledgments}

The authors would like to thank Aaron Sechler and Mike McMahon for their help during the initial planning phases of this project. Images were obtained from Wikimedia commons.

\section{Literature Cited}

Adams, I. P., Glover, R. H., Monger, W. A., Mumford, R., Jackeviciene, E., Navalinskiene, M., Samuitiene, M., and Boonham, N. 2009. Next-generation sequencing and metagenomic analysis: a universal diagnostic tool in plant virology. Mol. Plant Pathol. 10:537-545

Ammar, E. D., Shatters, R. G., Lynch, C., and Hall, D. G. 2011. Detection and relative titer of Candidatus Liberibacter asiaticus in the salivary glands and alimentary canal of Diaphorina citri (Hemiptera: Psyllidae) vector of citrus huanglongbing disease. Ann. Entomol. Soc. Am. 104:526-533.

Espindola, A., Schneider, W., Hoyt, P. R., Marek, S. M., and Garzon, C. 2015. A new approach for detecting fungal and oomycete plant pathogens in next generation sequencing metagenome data utilising electronic probes. Int. J. Data Min. Bioinform. 12:115-128.

Fagen, J. R., Giongo, A., Brown, C. T., Davis-Richardson, A. G., Gano, K. A., and Triplett, E. W. 2012. Characterization of the relative abundance of the citrus pathogen $\mathrm{Ca}$. Liberibacter asiaticus in the microbiome of its insect vector, Diaphorina citri, using high throughput 16S rRNA sequencing. Open Microbiol. J. 6:29-33.

García, J. A., Glasa, M., Cambra, M., and Candresse, T. 2014. Plum pox virus and sharka: a model potyvirus and a major disease. Mol. Plant Pathol. 15:226-241.

Gottwald, T. R. 2010. Current epidemiological understanding of citrus huanglongbing. Annu. Rev. Phytopathol. 48:119-139.

Jain, M., Fiddes, I. T., Miga, K. H., Olsen, H. E., Paten, B., and Akeson, M. 2015. Improved data analysis for the MinION nanopore sequencer. Nat. Methods 12 351-356.

Jiang, Z., Jones, D. H., Khuri, S., Tsinoremas, N. F., Wyss, T., Jander, G., Alex, C. C., and Wilson, A. C. C. 2013. Comparative analysis of genome sequences from four strains of the Buchnera aphidicola Mp endosymbion of the green peach aphid Myzus persicae. BMC Genomics 14:917.

Kilianski, A., Haas, J. L., Corriveau, E. J., Liem, A. T., Willis, K. L., Kadavy, D. R., Rosenzweig, C. N., and Minot, S. S. 2015. Bacterial and viral identification and differentiation by amplicon sequencing on the MinION nanopore sequencer. Gigascience 4:12.

Li, H., Handsaker, B., Wysoker, A., Fennell, T., Ruan, J., Homer, N., Marth, G., Abecasis, G., and Durbin, R. 2009. The sequence alignment/map format and SAMtools. Bioinformatics 25:2078-2079.

Morgan, M., Pagès, H., Obenchain, V., and Hayden, N. 2016. Rsamtools: Binary alignment (BAM), FASTA, variant call (BCF), and tabix file import. R package version 1.24.0. http://bioconductor.org/packages/release/bioc/html/Rsamtools.html

Nakabachi, A., Ueoka, R., Oshima, K., Teta, R., Mangoni, A., Gurgui, M. Oldham, N. J., van Echten-Deckert, G., Okamura, K., Yamamoto, K., and Inoue, H. 2013. Defensive bacteriome symbiont with a drastically reduced genome. Curr. Biol. 23:1478-1484.

Quick, J., Ashton, P., Calus, S., Chatt, C., Gossain, S., Hawker, J., Nair, S., Neal, K., Nye, K., Peters, T., De Pinna, E., Robinson, E., Struthers, K., Webber, M. Catto, A., Dallman, T. J., Hawkey, P., and Loman, N. J. 2015. Rapid draft sequencing and real-time nanopore sequencing in a hospital outbreak of Salmonella. Genome Biol. 16:114.

Quick, J., Quinlan, A. R., and Loman, N. J. 2014. A reference bacterial genome dataset generated on the MinIONportable single-molecule nanopore sequencer. Gigascience 3:22.

Quinlan, A. R., and Hall, I. M. 2010. BEDTools: a flexible suite of utilities for comparing genomic features. Bioinformatics 26:841-842.

Sović, I., Šikić, M., Wilm, A., Fenlon, S. N., Chen, S., and Nagarajan, N. 2016. Fast and sensitive mapping of nanopore sequencing reads with GraphMap. Nat. Commun. 7:11307.

Stobbe, A. H., Daniels, J., Espindola, A. S., Verma, R., Melcher, U., Ochoa-Corona, F., Garzon, C., Fletcher, J., and Schneider, W. 2013. E-probe diagnostic nucleic acid analysis (EDNA): a theoretical approach for handling of next generation sequencing data for diagnostics. J. Microbiol. Methods 94:356-366.

Stobbe, A. H., Schneider, W. L., Hoyt, P. R., and Melcher, U. 2014. Screening metagenomic data for viruses using the E-probe diagnostic nucleic acid assay. Phytopathology 104:1125-1129.

Ukuda-Hosokawa, R., Sadoyama, Y., Kishaba, M., Kuriwada, T., Anbutsu, H., and Fukatsu, T. 2015. Infection density dynamics of the citrus greening bacterium "Candidatus Liberibacter asiaticus" in field populations of the psyllid Diaphorina citri and its relevance to the efficiency of pathogen transmission to citrus plants. Appl. Environ. Microbiol. 81:3728-3736.

von Burg, S., Ferrari, J., Müller, C. B., and Vorburger, C. 2008. Genetic variation and covariation of susceptibility to parasitoids in the aphid Myzus persicae: no evidence for trade-offs. Proc. Royal Soc. B. 275:1089-1094.

Watson, M., Thomson, M., Risse, J., Talbot, R., Santoyo-Lopez, J., Gharbi, K., and Blaxter, M. 2014. poRe: an R package for the visualization and analysis of nanopore sequencing data. Bioinformatics 31:114-115. 